\title{
FREE-ELECTRON ANALYSIS OF OPTICAL PROPERTIES OF THERMALLY EVAPORATED GOLD FILMS
}

\author{
N. EL-KADRY \\ Pliysics Department, Minia University, El-Minia, Egypt
}

(Received November 3, 1993)

\begin{abstract}
Gold films of thicknesses 150-300 $\AA$ were deposited on quartz substrata using vacuum evaporation technique. Spectrophotometric measurements of transmission $T$ and reflection $R$ at normal incidence were performed in the range $0.4-3.0 \mathrm{fm}$. The real and imaginary parts of the complex refractive index $\tilde{n}$ were determined using a developed algoritlun based on Murmann's exact equations. The accuracy in the determined $n$ and $k$ was found to be $\pm 6.0 \%$ and $\pm 1.6 \%$, respectively. The clispersion curve of $n$ showed an anomalous dispersion in the visible region characterized by a peak at $\lambda=$ $0.840 \mathrm{~mm}$. The dielectric constants were calculated and presented. The Drude model parameters $\omega_{p}$ and $\omega_{\tau}$ and d.c. conductivity were determined and compared. The results showed that such parameters could be obtained from frce-clectron analysis for the near IR experimental results and the intraband transition contributes significantly to the diclectric functions.
\end{abstract}

PACS numbers: 78.66.13z, 78.30.Er

\section{Introduction}

The dispersion formula for intraband transition, as establishcd by Drude [1], treated the conduction clectrons as free particles of mass $m$ and charge $e$. The particles are accelerated by the electric field associaled with the incident wave and damped by a force proportional to their velocity, which is also responsible for their finite conductivity.

The Drude model is characterized by two parameters, namely $\omega_{p}$ and $\omega_{\tau}$ which are the plasma frequency of collective oscillations of the free electrons and the damping frequency, respectively. It was seen from earliest investigations that Drude's frcc-clectron theory failed in the visible and ultraviolet regions. In the noble metals, free-electron effects are dominant in IR region [2]. The intraband contribution to the dielectric constants can, therefore, be separated from the interband contribution by determining valucs for $\omega_{p}$ and $\omega_{\tau}$. On the other hand, 
Parkins et al. [3] showed that even the noble metals in the IR can have small interband contributions to the dielectric constants.

The most early determination of the diclectric constants as deduced from the optical constants are similar in their spectral beltaviour but disagree with their actual magnitude. Such disagreement is due to the problems of sample preparation, accuracy of measurements and method of determining the optical constants. In this regard, the Drude model, if it is appropriate, provides a useful parametrization for the optical constants data.

Bennet et al. [1] reported that the Drude model fits the measured reflectance of gold, silver and aluminium in the 3-30 $\mu \mathrm{m}$ wavelength range with one adjustable parameter, i.e., the Drude model parameters werc obtained from d.c. conductivity and fitted with one free electron per atom for gold and silver. Also it has been shown that [5] the Drude model provides a good fit for gold with no adjustable parameters in the far IR $(66.7 \mu \mathrm{m}$ to $318 \mu \mathrm{m})$.

Ordal et al. $[6,7]$ calculated $\omega_{\mathrm{p}}$ and $\omega_{\tau}$ which produced the curve with the best eyeball fit to the published cxpcrimental data, i.e., the values of $\omega_{\mathrm{p}}$ and $\omega_{\tau}$ obtained from the far IR data wcre changed by trial and error eyeball technique to obtain curves most closely matching the experimental data of $\varepsilon_{1}$ and $\varepsilon_{2}$. Also Ordal et al. [8] obtained values for the dielectric constants at submillimeter wavelengths using the Kramer-Kronig analysis of the normalized surface resistance determined from the non-resonant cavity measurements. They used a spline fit to bridge the data and they reported values for $\omega_{\tau}$ and $\omega_{p}$.

In this regard, it is noteworthy that such fitting techniques give a reasonable estimate for $\varepsilon_{1}$ but not for $\varepsilon_{2}$. In addition, the presence of a band structure combined with a lack of far IR data, limit the Drude model fit to a fairly narrow wavelength range (e.g., $\mathrm{Ti}$ ).

Several investigations of gold have used the Kramers-Kronig technique applied to normal-incidence reflection measurements. A problem of this method arises from the necessity of extrapolating the reflectance to frequencies outside the measured range. The choice of the extrapolation has a large effect on the magnitude of the optical constants, alliough the photon energies at which various structural features occur are insensitive to it.

In this work, the optical constants, $n$ and $k$, were accurately determined from spectrophotometric measurements of $R$ and $T$. A developed computing technique [9] based on Murmann's exact equations were used. The diclectric constants, $\varepsilon_{1}$ and $\varepsilon_{2}$, were calculated since they are closely related to the electronic structure of the solid and can be more directly compared with the theory. The free-electron analysis was performed in the near IR range $0.4-3 \mu \mathrm{m}$. Determination of the Drude model parameters was performed and the results are compared with those obtained through fitting techniques.

\section{Sample preparation and results}

Gold films were deposited on quartz substrata by thermal evaporation under vacuum better than $10^{-5} \mathrm{~mm} \mathrm{Hg}$. The substrata were cleaned ultrasonically and were dried by hot air. The substrata wcre masked until the source reached the 
evaporation temperature and thè rate of evaporation was made fast $\approx 70 \AA \mathrm{s}^{-1}$ to ensure the surface smoolhness of the films. The thickness of the films ranged from 150 to $300 \AA$ as determined in silu by a quartz thickness monitor. The transmission $T$ and reflection $R$ for light incident normally were measurcd at room temperature using a double beam spectrophotometer, UV-3101PC; Shimadzu. The values of $R$ and $T$ were corrected for reflection from the substrate surface. Figure 1 shows the spectral behaviour of the corrected values of $T$ and $R$ for films of different thicknesses. All films show high IR-reflectance increasing with film thickness. The curves show also a transmittance peak in the visible range which became more defined at ligher film thickness.

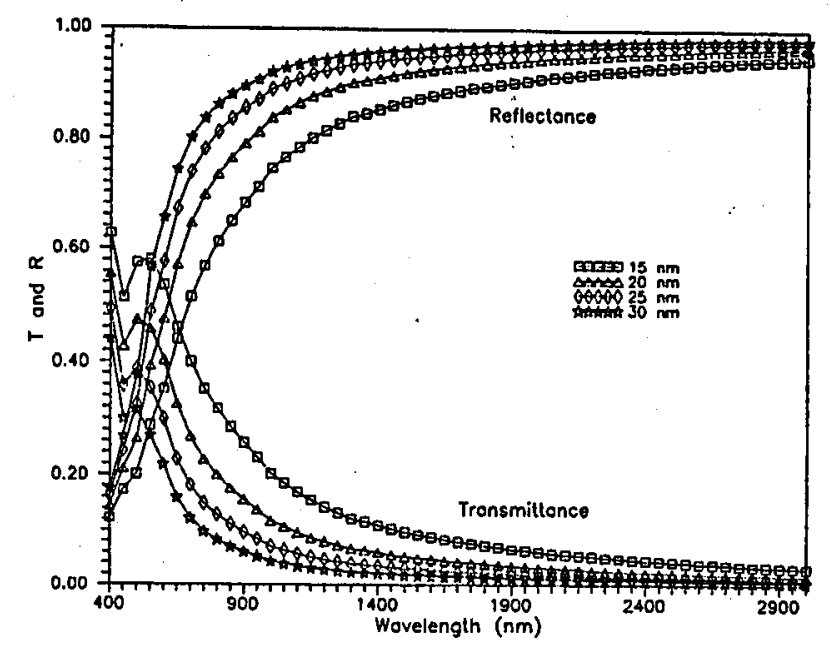

Fig. 1. Spectral variation of transmittance $T$ and reflectance $R$ of gold films.

The refractive index $n$ and the extinction index $k$ wcre determined over the spectral range from 0.4 to $3.0 \mu \mathrm{m}$ with $0.005 \mu \mathrm{m}$ wavelength step. The used method was previously 'explained [9] which is based on Murmann's exact equations. In Figs. 2 and 3 solid lines show the spectral variation obtained experimentally for $n$ and $k$, respectively. These values are averaged for several films. The height of the arrows represents the estimated error in calculating $n$ and $k$. Both errors get smaller towards the shorter wavelength. Figure 2 indicates that the refractive index $n$ exhibits anomalous dispersion in the visible region like other nobel metals [10]. Data given by other authors are represented for comparison $[8,11-13]$. It is clear that the spectral bchaviour of both $n$ and $k$ agree with one another while the same differences appear, as expected, between the absolute values, particularly in the $n$ values. The real and imaginary parts of the dielectric function were calculated and given in Table I. It is to be noted that the values of $n$ are small, in the IR region, while those of $k$ are large. Accordingly, the calculated values of $\varepsilon_{1}$ are relatively more accurate than those of $\varepsilon_{2}$ since the former is $\approx k^{2}$ while the latter is equal to $2 n k$. 
TABLE I

Optical and dielectric constants of gold films.

\begin{tabular}{c|r|c|c|c|c}
\hline \hline$\lambda[\mu \mathrm{m}]$ & $\omega\left[\mathrm{cm}^{-1}\right]$ & $n$ & $k$ & \multicolumn{1}{c|}{$-\varepsilon_{1}$} & \multicolumn{1}{c}{$\varepsilon_{2}$} \\
\hline 0.500 & 20000.000 & 0.730 & 2.000 & 3.467 & 2.920 \\
0.550 & 18181.818 & 0.345 & 2.650 & 6.903 & 1.829 \\
0.600 & 16666.666 & 0.295 & 3.100 & 9.523 & 1.829 \\
0.650 & 15384.616 & 0.277 & 3.650 & 13.246 & 2.022 \\
0.700 & 14285.715 & 0.258 & 4.150 & 17.156 & 2.141 \\
0.750 & 13333.333 & 0.260 & 4.600 & 21.092 & 2.392 \\
0.800 & 12500.000 & 0.250 & 5.000 & 24.938 & 2.500 \\
0.850 & 11764.705 & 0.252 & 5.400 & 29.096 & 2.722 \\
0.900 & 1111.111 & 0.255 & 5.800 & 33.575 & 2.958 \\
0.950 & 10526.316 & 0.267 & 6.200 & 38.369 & 3.311 \\
1.000 & 10000.000 & 0.270 & 6.680 & 44.550 & 3.607 \\
1.050 & 9523.810 & 0.270 & 7.060 & 49.767 & 3.897 \\
1.100 & 9090.909 & 0.285 & 7.450 & 55.421 & 4.247 \\
1.150 & 8695.652 & 0.295 & 7.850 & 61.535 & 4.631 \\
1.200 & 8333.333 & 0.305 & 8.230 & 67.610 & 5.020 \\
1.250 & 8000.000 & 0.313 & 8.610 & 74.034 & 5.390 \\
1.300 & 7692.308 & 0.323 & 9.000 & 80.896 & 5.814 \\
1.350 & 7407.407 & 0.335 & 9.300 & 86.378 & 6.231 \\
1.400 & 7142.857 & 0.343 & 9.650 & 93.005 & 6.620 \\
1.450 & 6896.551 & 0.353 & 10.000 & 99.875 & 7.060 \\
1.500 & 6666.667 & 0.365 & 10.350 & 106.989 & 7.556 \\
1.550 & 6451.613 & 0.375 & 10.680 & 113.922 & 8.010 \\
1.600 & 6250.000 & 0.385 & 11.000 & 120.852 & 8.470 \\
1.650 & 6060.606 & 0.397 & 11.350 & 128.665 & 9.012 \\
1.700 & 5882.353 & 0.410 & 11.670 & 136.021 & 9.569 \\
1.750 & 5714.286 & 0.420 & 12.000 & 143.824 & 10.080 \\
1.800 & 5555.556 & 0.435 & 12.320 & 151.593 & 10.718 \\
1.850 & 5405.405 & 0.445 & 12.650 & 159.824 & 11.258 \\
1.900 & 5263.158 & 0.462 & 13.000 & 168.787 & 12.012 \\
1.950 & 5128.205 & 0.475 & 13.300 & 176.664 & 12.635 \\
2.000 & 5000.000 & 0.490 & 13.660 & 186.355 & 13.387 \\
2.050 & 4878.049 & 0.503 & 14.000 & 195.717 & 14.084 \\
2.100 & 4761.905 & 0.515 & 14.300 & 204.225 & 14.729 \\
2.150 & 4651.163 & 0.525 & 14.630 & 213.761 & 15.361 \\
2.200 & 4545.455 & 0.510 & 14.960 & 223.510 & 16.157
\end{tabular}


TABLE I (cont.)

\begin{tabular}{c|c|c|c|c|c}
\hline \hline$\lambda[\mu \mathrm{m}]$ & $\omega\left[\mathrm{cm}^{-1}\right]$ & $n$ & $k$ & $-\varepsilon_{1}$ & $\varepsilon_{2}$ \\
\hline 2.250 & 4444.444 & 0.550 & 15.300 & 233.788 & 16.830 \\
2.300 & 4347.826 & 0.565 & 15.600 & 243.041 & 17.628 \\
2.350 & 4255.319 & 0.575 & 15.950 & 254.072 & 18.342 \\
2.400 & 4166.667 & 0.585 & 16.300 & 265.348 & 19.071 \\
2.450 & 4081.633 & 0.597 & 16.600 & 275.204 & 19.820 \\
2.500 & 1000.000 & 0.610 & 16.900 & 285.238 & 20.618 \\
2.550 & 3921.569 & 0.620 & 17.260 & 297.523 & 21.402 \\
2.600 & 3846.154 & 0.635 & 17.600 & 309.357 & 22.352 \\
2.650 & 3773.585 & 0.645 & 17.950 & 321.787 & 23.156 \\
2.700 & 3703.704 & 0.657 & 18.200 & 330.808 & 23.915 \\
2.750 & 3636.364 & 0.670 & 18.650 & 347.347 & 24.991 \\
2.800 & 3571.429 & 0.680 & 18.900 & 356.748 & 25.704 \\
2.850 & 3508.772 & 0.690 & 19.230 & 369.317 & 26.537 \\
2.900 & 3448.276 & 0.708 & 19.560 & 382.092 & 27.697 \\
2.950 & 3389.831 & 0.720 & 19.900 & 395.492 & 28.656 \\
3.000 & 3333.333 & 0.736 & 20.200 & 407.498 & 29.734
\end{tabular}

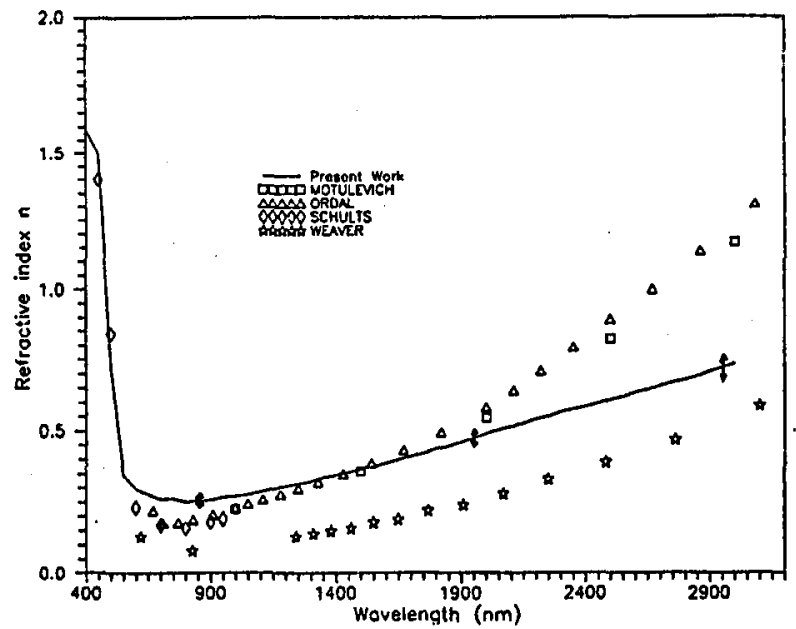

Fig. 2. Spectral variation of the real refractive index $n$. 


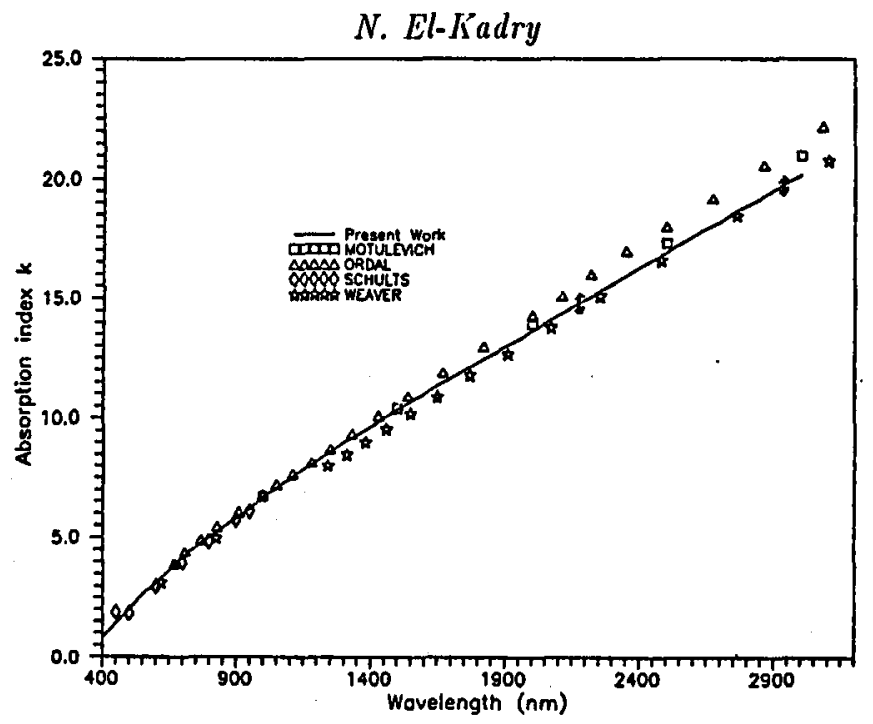

Fig. 3. Spectral variation of the absorption index $k$.

\section{Detcrmination of the Drude model parameters}

If $n$ and $k$ are the real and imaginary parts of the complex refractive index $\tilde{n}$, then Drude's theory yields the following expressions for the dielectric functions:

$$
\tilde{\varepsilon}=\varepsilon_{1}+\mathrm{i} \varepsilon_{2}=(n+\mathrm{i} k)^{2}=\varepsilon_{00}-\frac{\omega_{\mathrm{p}}^{2}}{\omega^{2}+\mathrm{i} \omega \omega_{\tau}},
$$

where $\varepsilon$ is the high frequency dielectric constant, $\omega_{\mathrm{p}}$ and $\omega_{\tau}$ are the plasma and damping frequencies expressed in $\mathrm{cm}^{-1}$.

Separating real and imaginary parts we get

$$
\varepsilon_{1}=\varepsilon_{00}-\frac{\omega_{\mathrm{p}}^{2}}{\omega^{2}+\omega_{\tau}^{2}}, \quad \varepsilon_{2}=\frac{\omega_{\tau} \omega_{\mathrm{p}}^{2}}{\omega\left(\omega_{\tau}^{2}+\omega^{2}\right)} .
$$

Solving Eq. (2) for $\omega_{\mathrm{p}}$, we get

$$
\omega_{\tau}=\frac{\omega \varepsilon_{2}}{1-\varepsilon_{1}} \text { then } \omega_{\mathrm{p}}=\left(1-\varepsilon_{1}\right)\left(\omega^{2}+\omega_{\tau}^{2}\right) .
$$

The last two equation were used previously to calculate the lowest frequency (far IR) data for $\omega_{\tau}$ and $\omega_{\mathrm{p}}[6-8]$ then changed through $\varepsilon_{1}$ and $\varepsilon_{2}$ by trial and error to obtain curves most closely matching the experimental data for $\varepsilon_{1}$ and $\varepsilon_{2}$ in both behaviour and magnitude.

In this work the Drude parameters were determined directly from the results obtained experimentally for the optical constants $n$ and $k$ as follows.

Rearranging the terms in Eqs. (2) we get

$$
\frac{1}{1-\varepsilon_{1}}=\frac{\omega^{2}+\omega_{\tau}}{\omega_{\mathbf{p}}^{2}}
$$

from which we get

$$
\frac{1}{\omega^{2}} \frac{1}{\left(1-\varepsilon_{1}\right)}=\frac{\omega_{\tau}}{\omega_{\mathrm{p}}^{2}} \frac{1}{\omega^{2}}+\frac{1}{\omega_{\mathrm{p}}^{2}}
$$




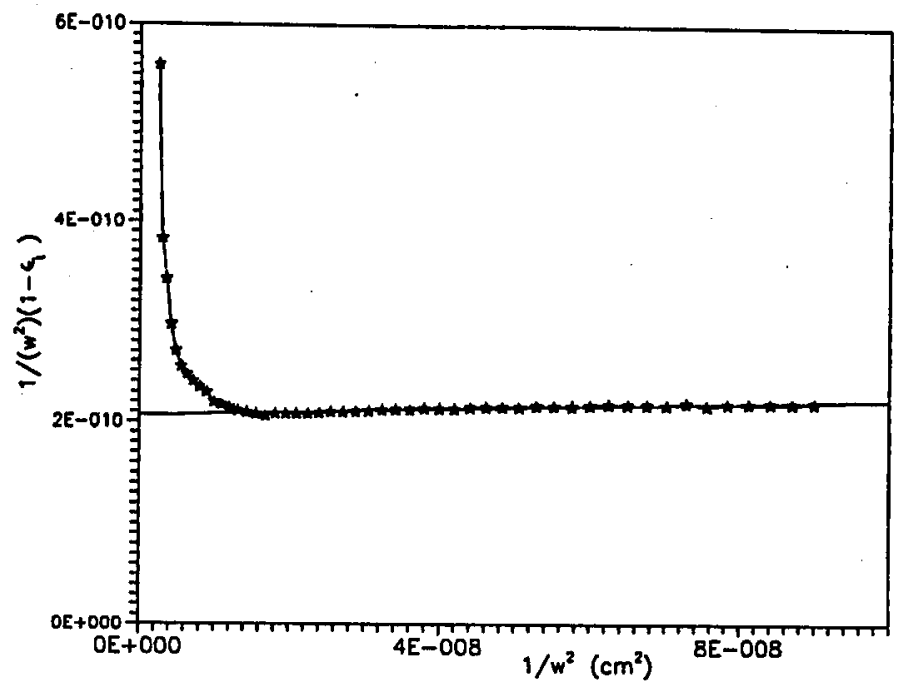

Fig. 4. Plot of $\left[\omega^{2}\left(1-\varepsilon_{1}\right)\right]^{-1}$ vs. $1 / \omega^{2}$.

The last equation shows that the plot of $1 / \omega^{2}\left(1-\varepsilon_{1}\right)$ vs. $1 / \omega^{2}$ is linear as required by free-electron theory. The Drude model parametcrs are obtained from the slope of the straight line and its intercept witl the ordinate.

Figure 4 shows a plot of $1 / \omega^{2}\left(1-\varepsilon_{1}\right)$ vs. $1 / \omega^{2}$ based on the determined values of $n$ and $k$ obtained experimentally. It is clear that for $\lambda>1.2 \mu \mathrm{m}$, a linear relation resulted as required by the free-clectron theory. Thus, it is clear that for $\lambda>1.2 \mu \mathrm{m}$ intraband transition contributes significantly throughout the spectral range investigated in this work. This means that the free-electron beliaviour dominates in the near IR where $n$ is small and $k$ is large. From the intercept at the ordinate, the plasma frequency $\omega_{\mathrm{p}}$ was calculated and found to be $6.69 \times 10^{4} \mathrm{~cm}^{-1}$ whereas from its slope, the damping frequency was found to be $2.92 \times 10^{2} \mathrm{~cm}^{-1}$. Comparison between the present results and those obtained by other authors through fitting techniques is given in Table II. It is clear that our values are more adjacent to the values reported recently and obtained by analysis of non-resonant cavity measurements of surface resistance.

The high frequency optical conductivity $\sigma_{\text {opt }}$ is related to $\omega_{\mathrm{p}}$ and $\omega_{\tau}$ by the relation [7]:

$$
\sigma_{\mathrm{opt}}\left[\mathrm{cm}^{-1}\right]=\frac{\omega_{\mathrm{p}}^{2}}{2 \pi \omega_{\tau}}=\frac{9 \times 10^{11}}{2 \pi c\left(\rho_{\mathrm{opt}}[\Omega \mathrm{cm}]\right)} .
$$

Substituting for $\omega_{\mathrm{p}}$ and $\omega_{\tau}$ yielded the optical resistivity: $\rho_{\mathrm{opt}}=3.6 \times 10^{-6} \Omega \mathrm{cm}$. This value is slightly higher than the d.c. resistivity given in text books for bulk, namely, $\rho_{0}=2.44 \times 10^{-6} \Omega \mathrm{cm}$. It is to be noted that our value was obtained for films, $d \approx 200 \AA$, which is not necessarily representing the bulk. 
TABLE II

Results of Drude model parameters for gold.

\begin{tabular}{l|l|l}
\hline \hline \multicolumn{1}{c|}{ Author } & $\omega_{\tau}\left[\mathrm{cm}^{-1}\right]$ & $\omega_{\mathrm{p}}\left[\mathrm{cm}^{-1}\right]$ \\
\hline $\begin{array}{l}\text { Ordal ct al. [6, 7] } \\
\text { using far IR fit }\end{array}$ & $2.15 \times 10^{2}$ & $7.28 \times 10^{4}$ \\
& & \\
$\begin{array}{l}\text { Ordal ct al. [8] } \\
\text { using surface resistance } \\
\text { fit at submillimeter range }\end{array}$ & $3.00 \times 10^{2}$ & $7.25 \times 10^{4}$ \\
& & \\
Prescnt work & $2.92 \times 10^{2}$ & $6.97 \times 10^{4}$
\end{tabular}

The optical mass $m_{0}$ and the relaxation time $\tau$ are related to the Drude parameters by the relations

$$
\omega_{\mathrm{p}}^{2}=\frac{4 \pi N e^{2}}{m_{0}} \quad \text { and } \quad \omega_{\tau}=\frac{1}{2 \pi c \tau}
$$

where $N$ is the density of the conduction electrons. Substituting for $\omega_{\mathrm{p}}=6.69 \times$ $10^{4} \mathrm{~cm}^{-1}$ and $\omega_{\tau}=2.92 \times 10^{2} \mathrm{~cm}^{-1}$, we get $m_{0}=10.3 \times 10^{-27} \mathrm{~kg}=1.1 \mathrm{~m}_{\mathrm{e}}$ and $\tau=18 \times 10^{-15} \mathrm{~s}$.

\section{Conclusion}

The optical constants of thermally evaporated gold films are presented over the spectral range from $0.1-3.0 \mu \mathrm{m}$. Frec-electron analysis showed that intraband transition contributes significantly to the diclectric constants. The Drude model parameters could be successfully obtained directly from the near IR data, i.e., the calculated values are in good agrecment with those determined through fitting techniques of data obtained by either far IR or normalized surface resistance measurcments.

\section{References}

[1] F. Wooten, Oplical Properlies of Solids, Academic, New York 1972, p. 52.

[2] P.B. Johnson, R.W. Clıristy, Phys. Rev. B G, 4370 (1972).

[3] G.R. Parkins, W.E. Lawrence, R.W. Christy. Phys. Rev. B 23, 6408 (1981).

[4] II.E. Bennet, J.M. Bennet, in: Optical Properties and Eleclronic Structure of Metals and Alloys, Ed. F. Abeles, North-IIolland, Amsterdim, Wiley, New York 1966, Sec. 1I.6, p. 175.

[5] G. Brandi, A.J. Sievers, Phys. Rev. B 5, 3550 (1972).

[6] M.A. Ordal, L.L. Long, R.J. Bell, S.E. Bell, R.R. Bell, R.W. Alexander Jr., C.A. Ward, Appl. Opt. 22, 1099 (1983). 
[7] M.A. Orda], R.J. Bell, R.W. Alexantler Jr., L.L. Long, M.R. Qerry, Appl. Opt. 24; 4493 (1985).

[8] M.A. Ordal, R.J. Bell, R.W. Alexander Jr., J.L. Long, M.R. Querry, Appl. Opt. 26, 744 (1987).

[9] M.M. El-Nahass, II.S. Soliman, N. El-Kadry, A.Y. Morsy, S. Yaghımour, J. Mater. Sci. Lelt. 7, 1050 (1988).

[10] N. El-Kadry, F. Kaid, Egypt. J. Sol. 15, 9 (1992).

[11] L.G. Scultz, J. Opt. Soc. Am. 44, 357 (1954).

[12] G.P. Motulevich, A.A. Shubiı, Sov. Phys. JETP 20, 560 (1965).

[13] T.II. Wea ver, C. Krafka, D.W. Lyıch, E.E. Koch, Physics Dala, Optical Properties of Melals, Fachinformation Zentrum, Karlsruhe 1981. 